\title{
Quantitative comparison of left ventricular motion parameters for the assessment of asynchrony and motion abnormalities
}

\author{
Raphael Beck', Jan Paul ${ }^{1 *}$, Anja Müller-Lutz ${ }^{1,2}$, Dominik Buckert ${ }^{1}$, Peter Bernhardt', Wolfgang Rottbauer ${ }^{1}$, \\ Volker Rasche ${ }^{1}$
}

From 17th Annual SCMR Scientific Sessions

New Orleans, LA, USA. 16-19 January 2014

\section{Background}

Different motion parameters for quantification of heart motion abnormalities and asynchrony have been described in literature. The aim of this study is the direct comparison of reported parameters in different patient groups.

\section{Methods}

Cohorts and Acquisition: 41 volunteers (HV, $25 \pm 5$ y.), 14 STEMI (63 \pm 7 y.), 12 DCM $(54 \pm 17$ y.), and 5 DCM+LBBB ( $47 \pm 8$ y.) patients were investigated. Acquisition parameters were: Philips Achieva $3 \mathrm{~T}$, 32 channel cardiac coil, velocity encoded (Tissue Phase Mapping, TPM) segmented black-blood gradient echo with VENC $=30 \mathrm{~cm} / \mathrm{s}, \mathrm{TR} / \mathrm{TE}=6.1 / 4.6 \mathrm{~ms}$, FOV adapted to patient size, resolution $=2^{2} \times 8 \mathrm{~mm}^{3}$, $3 \mathrm{k}$-lines $/$ segment, SENSE $=2$, phase interval $=30 \mathrm{~ms}$, and nominal scan time $=5: 51 \mathrm{~min}: \mathrm{sec}$ for 3 short axis slices. Parameters (see [1]): a) velocity-based: Standard Deviation of Times to Peak [SD(TTP)], Asynchrony Correlation Coefficient [ACC], Temporal Uniformity of Velocity [TUV], and Velocity Ranges (difference: maximum-minimum velocity). b) strain-based: Base Apex Rotation Correlation [BARC], Temporal Uniformity of Strain [TUS], Standard Deviation of Onset/Peak Time $[\mathrm{SD}(\mathrm{T})]$, Coefficient of Variation [CV], Difference between Septal and Lateral Peak Circumferential Strain [DiffSLpeakCS], Onset/Peak Of Shortening Delay [Delay], Regional Variance of Strain [RVS], and Regional Variance Vector of Strain [RVVPS]. Analysis: The significance of resulting differences between the different

${ }^{1}$ Internal Medicine II, University Hospital of UIm, Ulm, Germany Full list of author information is available at the end of the article groups was assessed via non-parametric Kruskal-WallisTest.

\section{Results}

Statistics of comparison between the different cohorts are presented in table 1. Velocity-based: Velocity ranges are significantly reduced in HV and patients with structural heart disease, especially in radial and longitudinal direction over all three short-axis slices (see Figure 1). $\mathrm{SD}$ (TTP) for diastolic, longitudinal velocity is increased in all patients; $\mathrm{SD}$ (TTP) for systolic, radial velocity is significantly higher in patients with DCM+LBBB. ACC (longitudinal) is significantly decreased in patient cohorts compared to HV. ACC(radial, average/maximum) separates LBBB from all other cohorts. Strain-based: BARC highlights loss of torsion in patients with DCM and LBBB. TUS, RVS, and RVVPS are able to detect dyssynchrony in $\mathrm{LBBB} . \mathrm{SD}(\mathrm{T}$, peak) is raised in patients. $\mathrm{SD}(\mathrm{T}$, onset) indicates asynchrony in LBBB patients. CV shows significant increase in LBBB vs. all other cohorts. In this study DiffSLpeakCS is not capable to make distinction between HV and patients. Delay(peak, septal-lateral/ inferior-anterior) show characteristic aberrations for STEMI and LBBB compared to HV.

\section{Conclusions}

Most distinct results were found in velocity ranges, indicating damaged myocardium, although they featured no particular value for differentiation between LBBB and other patient cohorts. Parameters calculated on the entire cardiac cycle in each slice (velocity and strain: ACC, TUS, RVS, RVVPS) proved capable of detecting ventricular asynchrony due to LBBB 
Table 1 Comparison of parameter differences between different cohorts: not significant $(-), p<5 \%(*), p<1 \%(* *), p<0.1 \%\left({ }^{* * *}\right)$.

\begin{tabular}{|c|c|c|c|c|c|c|c|c|c|}
\hline \multicolumn{5}{|c|}{ Parameter } & \multicolumn{3}{|c|}{ HV vs. } & \multicolumn{2}{|c|}{ LBBB vs. } \\
\hline & & & & & STEMI & DCM & LBBB & STEMI & DCM \\
\hline \multirow[t]{4}{*}{ velocity based } & . & o & $\mathrm{SD}(\mathrm{TTP})$ & $\begin{array}{c}\text { systolic, radial } \\
\text { diastolic, radial } \\
\text { systolic, longitudinal } \\
\text { diastolic, longitudinal }\end{array}$ & $\begin{array}{c}- \\
- \\
* * \\
* * * \\
\end{array}$ & $\begin{array}{c}- \\
- \\
- \\
* * * \\
\end{array}$ & $\begin{array}{c}* * * \\
* * \\
* \\
* * * \\
\end{array}$ & $\begin{array}{l}* * \\
- \\
- \\
* \\
\end{array}$ & $\begin{array}{c}* * \\
- \\
- \\
* \\
\end{array}$ \\
\hline & / & - & ACC & $\begin{array}{c}\text { radial, minimum } \\
\text { radial, average } \\
\text { radial, maximum } \\
\text { circumferential, minimum } \\
\text { circumferential, average } \\
\text { circumferential, maximum } \\
\text { longitudinal, minimum } \\
\text { longitudinal, average } \\
\text { longitudinal, maximum }\end{array}$ & $\begin{array}{l}* \\
- \\
- \\
- \\
- \\
* \\
* * \\
* * * \\
* *\end{array}$ & $\begin{array}{c}* \\
* \\
- \\
* * \\
* \\
- \\
* \\
* * \\
* * *\end{array}$ & $\begin{array}{l}* * * \\
* * * \\
* * * \\
* \\
* \\
- \\
* * \\
* * * \\
* * *\end{array}$ & $\begin{array}{l}- \\
* * \\
* * \\
- \\
* \\
- \\
- \\
* \\
*\end{array}$ & $\begin{array}{c}* * \\
* * \\
* * * \\
* * \\
- \\
- \\
- \\
- \\
* * \\
* *\end{array}$ \\
\hline & / & - & TUV & $\begin{array}{c}\text { radial } \\
\text { circumferential } \\
\text { longitudinal }\end{array}$ & $\begin{array}{c}- \\
- \\
- \\
* * *\end{array}$ & $\begin{array}{l}- \\
* \\
*\end{array}$ & $\begin{array}{l}* * * \\
* * \\
* * *\end{array}$ & $\begin{array}{l}\text { ** } \\
- \\
* *\end{array}$ & $\begin{array}{l}* \\
- \\
*\end{array}$ \\
\hline & / & & Velocity Range & $\begin{array}{c}\text { longitudinal, apical } \\
\text { longitudinal, equatorial } \\
\text { longitudinal, basal } \\
\text { circumferential, apical } \\
\text { circumferential, equatorial } \\
\text { circumferential, basal } \\
\text { radial, apical } \\
\text { radial, equatorial } \\
\text { radial, basal }\end{array}$ & $\begin{array}{c}* * \\
* * * \\
* * * \\
- \\
* * * \\
* * * \\
* * * \\
* * * \\
* * *\end{array}$ & $\begin{array}{l}* * * \\
* * * \\
* * * \\
* * * \\
* * * \\
* * \\
* * * \\
* * * \\
* * *\end{array}$ & $\begin{array}{l}* * * \\
* * * \\
* * * \\
* * \\
* \\
- \\
* * * \\
* * * \\
* * *\end{array}$ & $\begin{array}{l}- \\
- \\
- \\
* \\
- \\
- \\
- \\
* \\
-\end{array}$ & $\begin{array}{l}- \\
- \\
- \\
- \\
- \\
- \\
- \\
- \\
-\end{array}$ \\
\hline \multirow[t]{9}{*}{ position/strain based } & I & | & BARC & & - & - & ** & * & * \\
\hline & I & - & TUS & $\begin{array}{l}\text { circumferential } \\
\text { radial }\end{array}$ & $\begin{array}{l}- \\
-\end{array}$ & $\begin{array}{l}- \\
-\end{array}$ & $\begin{array}{l}* * * \\
* *\end{array}$ & $\begin{array}{l}* * \\
* *\end{array}$ & $\begin{array}{l}* \\
* * \\
* *\end{array}$ \\
\hline & I & - & RVS & & - & $*$ & - & $*$ & $* * *$ \\
\hline & 1 & - & RWPS & & - & - & ** & ** & *** \\
\hline & . & 0 & $\mathrm{SD}(\mathrm{T})$ & onset & - & * & ** & ** & * \\
\hline & 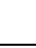 & & & peak & $* * *$ & $* * *$ & $* * *$ & - & * \\
\hline & . & o & $\mathrm{CV}$ & & - & - & $* * *$ & $* *$ & $* *$ \\
\hline & . & - & DiffSLpeakCS & & - & - & - & - & - \\
\hline & . & $\begin{array}{l}- \\
- \\
1 \\
- \\
\end{array}$ & Delay & $\begin{array}{l}\text { onset, septal-lateral } \\
\text { onset, inferior-anterior } \\
\text { onset, apical-basal } \\
\text { peak, septal-lateral } \\
\text { peak, inferior-anterior } \\
\text { peak, apical-basal }\end{array}$ & $\begin{array}{c}- \\
- \\
- \\
* * \\
* * * \\
*\end{array}$ & $\begin{array}{l}* \\
- \\
* * \\
- \\
- \\
-\end{array}$ & $\begin{array}{c}* \\
- \\
- \\
* * * \\
* * * \\
* *\end{array}$ & $\begin{array}{l}- \\
- \\
- \\
* \\
- \\
-\end{array}$ & $\begin{array}{l}- \\
- \\
- \\
* \\
* \\
-\end{array}$ \\
\hline
\end{tabular}




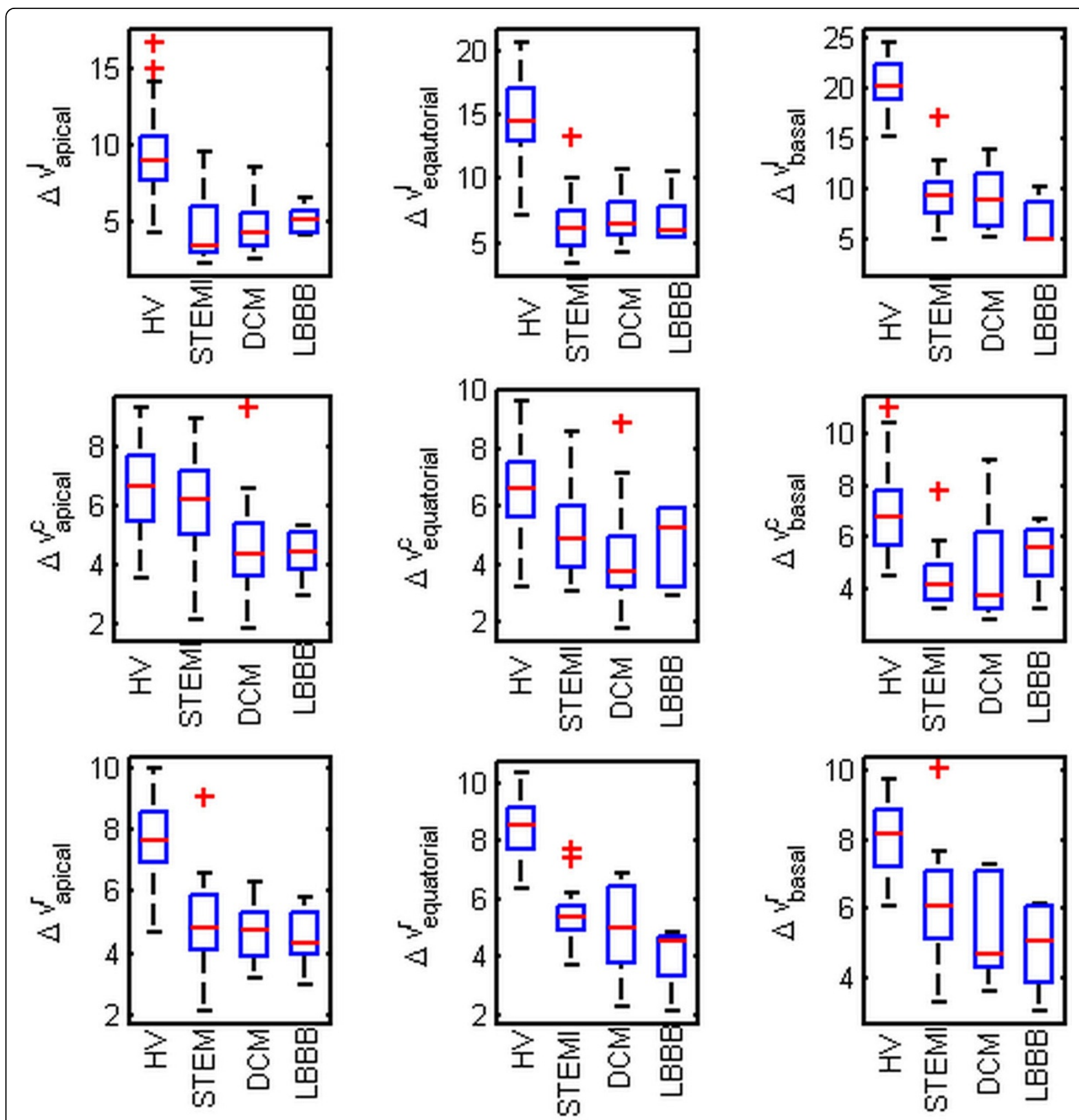

Figure 1 Distribution of velocity ranges $\left(\Delta v=v_{\max }-v_{\min }\right)$ in radial ( $\left.r\right)$, circumferential (c) and longitudinal (I) direction for apical, equatorial and basal slices. Ranges are decreased in all patient cohorts compared to volunteers.

\section{Funding}

None.

\section{Authors' details}

'Internal Medicine II, University Hospital of Ulm, Ulm, Germany. ${ }^{2}$ Department of Diagnostic and Interventional Radiology, University of Dusseldorf, Medical Faculty, Dusseldorf, Germany.
Reference

1. Lutz, et al: JCMR 2012, 14:74

doi:10.1186/1532-429X-16-S1-P94

Cite this article as: Beck et al:: Quantitative comparison of left

ventricular motion parameters for the assessment of asynchrony and

motion abnormalities. Journal of Cardiovascular Magnetic Resonance 2014

16(Suppl 1):P94. 